\title{
Pertenencia inicial de descendientes a la empresa familiar
}

\author{
Lozano P., Melquicedec* \\ Urbano, David**
}

\section{Resumen}

En el contexto de la sucesión, el presente estudio de casos examina la pertenencia inicial de los descendientes a las empresas familiares colombianas. Adicionalmente se exponen las principales proposiciones que relacionan, satisfacción y compromiso, con ese primer trabajo a tiempo completo en la empresa familiar. La metodología considera entrevistas en profundidad a descendientes de cuatro casos de empresas familiares, cuya información se somete posteriormente a un análisis cualitativo apoyándose en el concepto de triangulación y la teoría de recursos y capacidades. Los resultados del estudio resaltan la satisfacción y el compromiso como los dos componentes de la pertenencia inicial; igualmente, se determinan los ítems que conforman cada uno de estos componentes, lo que amplía conceptualmente el panorama relacionado con la continuidad de las empresas familiares. Las conclusiones muestran que la propensión a tener más satisfacción y compromiso con el primer cargo está muy relacionada con el tipo de ambiente que perciben los descendientes y con la posibilidad de satisfacer sus motivaciones.

Palabras clave: Empresa familiar, sucesión, satisfacción, compromiso, Colombia.

\section{Recibido: 20-01-10. Aceptado: 15-05-10}

Director de Investigaciones y Proyectos Especiales, Centro de Desarrollo del Espíritu Empresarial. Universidad Icesi, Cali, Colombia. melqui@icesi.edu.co.

** Subdirector Doctorado European Doctoral Programme in Entrepreneurship and Small Business Management, Departamento de Economía de la Empresa. Universidad Autónoma de Barcelona. España. david.urbano@uab.es. 


\title{
First Full-Time Job of Descendents in the Family Business. A Qualitative Study in Colombia
}

\begin{abstract}
In the context of succession, this Colombian case study examines the first full-time job that descendents have in their family's business. Furthermore, it describes the main propositions -satisfaction and commitment- that relate to this first full-time job in the family business. The methodology includes in-depth interviews with descendents from four family business cases; the information was later submitted to qualitative analysis, based on the triangulation concept and the resources and capacities theory. Results of the study highlight satisfaction and commitment as two components of the initial membership; likewise, items that constitute each of these components were determined, thereby extending, conceptually, the panorama related to the continuity of family businesses. In conclusion, this study shows that the propensity to experience more satisfaction and commitment with the first full-time job is quite related to the type of environment the descendants perceive and the possibility of satisfying their motivations.
\end{abstract}

Key words: Family business, succession, satisfaction, commitment, Colombia.

\section{Introducción}

Por su alto porcentaje en el total de empresas y por su gran aporte al producto interno bruto, la empresa familiar se considera de gran importancia en la economía de un país con sistema de libre mercado (Neubauer y Lank, 1999). Además, dada su condición en la cual la familia propietaria tiene el control de la propiedad y, con frecuencia, está involucrada en la administración de la misma, la empresa familiar continúa siendo un área de sumo interés para los estudiosos de las organizaciones, siendo reconocida como un campo de gran amplitud para la investigación presente y hacia el futuro (Gimeno et al., 2008), por lo cual, los trabajos empíricos en el presente siglo XXI se han incrementado.

En el campo de las empresas familiares hay muchas áreas que se han investigado, Sin embargo, la sucesión sigue erigiéndose como uno de los temas trascendentales en el campo, por lo cual todas las etapas del proceso de sucesión toman gran trascendencia. En este proceso continuo de sucesión se hace muy importante la formación que se le da al potencial sucesor en toda la etapa previa a la entrada a la empresa (Ward, 1994; Morris et al., 1996 y 1997; Cabrera et al., 2001). Posterior a esta etapa de formación (Lozano, 2008) se identifica un corto período denominado etapa de vinculación (Harvey y Evans, 1994; Lozano y Urbano, 2008), que precede luego a la etapa inicial de pertenencia a la empresa familiar o primer trabajo a tiempo completo.

En esta investigación se explora esta etapa, es decir, lo que vivieron los hijos, o los hijos de hijos, actualmente vinculados, en su primer trabajo de tiempo completo en la empresa familiar.

Si en esta etapa el descendiente se siente satisfecho con lo que hace, y además cumple de manera responsable con las funciones, es bastante probable que 
continúe en la compañía, haciendo con ello más favorable al tema de continuidad de la empresa familiar. Estos dos factores se traducen en satisfacción y compromiso, factores que han sido estudiados en la literatura sobre empresas familiares, pero sin la preocupación por estudiar el fenómeno en la etapa inicial de trabajo a tiempo completo. Es decir, vistos los dos, satisfacción y compromiso, como factores que componen el concepto pertenencia a la organización. La pregunta que nos compete aquí es ¿cómo se manifiesta la pertenencia con el primer cargo asumido a tiempo completo, y qué proposiciones se desprenden de ello? Por lo tanto, el objetivo fundamental de este estudio exploratorio es definir los constructos de los factores satisfacción y compromiso como pilares de la primera fase de pertenencia a la empresa familiar colombiana, y formular proposiciones que puedan ser contrastadas en estudios cuantitativos posteriores.

En cuanto a la metodología, el estudio se apoya en el análisis de descendientes vinculados a cuatro casos de empresas familiares colombianas. Para abordar la investigación fue oportuno contar con una definición de lo que es una empresa familiar, ya que existen muchas definiciones (Davis, 1983: 47; Wortman, 1994; Westhead, 1997; Westhead y Crowling, 1998; Winter et al., 1998; Chua et al., 1999; Upton et al., 2001:61; Astrachan y Shanker, 2003) y no hay una única definición, pero parece haber acuerdo en cuanto que las tres dimensiones fundamentales que contribuyen a su definición son: la propiedad/dirección de los miembros de una familia, la implicación familiar, la transferencia generacional. Por consiguiente, se adoptó con leves ajustes la definición dada por cabrera (2005), que cumple con estas dimensiones. Por lo tanto, la definición asumida es la siguiente: una empresa familiar es aquella en que el $51 \%$ o más de la propiedad está en manos de los miembros de una familia, que tienen la intención de que las relaciones intraempresariales de propiedad y control directivo estén basadas en lazos familiares y en donde se ha incorporado la segunda generación o posteriores. Para efectos de los objetivos planteados aquí, se asume como segunda generación o posteriores, a los hijos descendientes del gestor o gestores, y su correspondiente descendencia de hijos a hijos, a los cuales llamamos aquí descendientes o potenciales sucesores.

Se eligió el estudio de casos para esta investigación dado que la principal pregunta del problema de investigación gira en torno del cómo y porqué, además, se busca determinar la relación que tienen los factores con la pertenencia a la empresa, así como nuevos conceptos que permitan avanzar en el conocimiento del fenómeno, lo que, de acuerdo a Yin (1994), se ajusta al diseño de una investigación cualitativa. En consecuencia, se utilizaron múltiples fuentes de evidencia necesarias para triangular la información, teniendo el marco teórico y la pregunta de investigación como punto de partida.

Como en la literatura no hay definiciones para la satisfacción y el compromiso de los descendientes en el primer cargo de tiempo completo en la empresa familiar, se asume aquí, con base en los trabajos de Sharma e Irving (2005) y Sharma et al. (2001 y 2003a), las definiciones ajustadas. Satisfacción: se refiere a los 
sentimientos de agrado con las experiencias que se viven durante el desempeño del primer trabajo a tiempo completo. Compromiso: se refiere a la fuerza del descendiente que lo induce a acciones relevantes para alcanzar objetivos de beneficio para la empresa familiar, durante ese primer trabajo.

En esta investigación los cuatro casos elegidos cumplen con los siguientes criterios de selección previamente establecidos: ser empresas medianas o grandes, tener al menos diez años de existencia, que tuvieran vinculados miembros de la segunda generación o posteriores, que de la última generación vinculada existiese al menos un miembro familiar trabajando de tiempo completo en la empresa.

La selección de los cuatro casos partió de un listado de empresas familiares ya existente y se tomó el mínimo aconsejable para el estudio cualitativo (Lambrech, 2005: 270; Eisenhardt, 1989:545), aunque no hay una guía precisa acerca del número de casos (Perry, 1998).

Para el proceso de recolección de datos se tuvo en cuenta que Yin (1994) recomienda la utilización de múltiples fuentes de datos y el cumplimiento del principio de triangulación para garantizar la validez interna de la investigación. Al efecto, las fuentes de información utilizadas en este estudio fueron: las entrevistas a descendientes de la última generación vinculada, las entrevistas a familiares de los descendientes, las visitas realizadas a las instalaciones de las empresas, los documentos proporcionados por éstas.

En cuanto al criterio de fiabilidad, en esta investigación se elaboró un proto- colo de estudio y se desarrolló una base de datos de las trascripciones de las entrevistas y de los textos relevantes seleccionados, que puede ser consultada en la unidad hermenéutica del ATLAS/ti 5.0, programa de ayuda al análisis cualitativo de datos, que fue utilizado por los investigadores.

Para el análisis de la información se tuvo en cuenta las recomendaciones acerca de hacer un análisis inductivo de los datos (Marshall y Rossman, 1995) guiado por el marco teórico de la investigación, aunque también se tuvo en cuenta las anotaciones de Miles y Huberman (1994), que sugiere un análisis deductivo de la información con apoyo de categorías y códigos. Se utilizó por tanto, una metodología iterativa mediante la cual, partiendo del marco teórico y la pregunta de investigación se leyeron y releyeron las trascripciones de las entrevistas y notas de campo, con lo cual se construyeron las categorías y códigos de la información (Strauss y Corbin, 1990; Miles y Huberman, 1994).

El proceso iterativo confirmó la existencia de los dos pilares de la estructura de la pertenencia a la empresa, a saber, satisfacción y compromiso, No se evidenciaron factores adicionales, pero sí se avanzó en la composición de cada uno de estos factores y en las proposiciones que de ello se derivaron, tal como se muestra más adelante en el numeral 3.

El estudio se aborda bajo la teoría de recursos y capacidades (Wernelfelt, 1984; Diericx y Cool, 1989; Barney, 1991; Peteraf, 1993), pues ella establece que la consecución de resultados superiores por parte de las empresas, radica en que estas disponen y controlan recursos críti- 
cos y capacidades especiales que las diferencian de las demás; rasgos que son habituales en el contexto de personas propietarias o de descendientes de empresas familiares.

\section{Literatura sobre sucesión}

Muchos estudios tratan la sucesión en empresas familiares (Longenecker y Schoen, 1978; Handler y Kram, 1988; Wortman, 1994; Ussman, 1994; Sharma, 2003a y 2003b; Venter et. al., 2005; Cadieux, 2007; Haberman y Danes, 2007), y hoy los temas de empresa familiar y sucesión continúan siendo un par de siameses que andan siempre juntos (Lambrech, 2005: 267-268); lo que estimula a seguir ahondando sobre este campo. Se argumenta que la planeación de la sucesión debería ser vista como un proceso de largo plazo que comienza mucho antes de que los descendientes entren a la compañía (Stavrou y Swiercz, 1998:20). Sin embargo, hay pocas investigaciones sobre procesos de preparación de los hijos antes de su incorporación a la empresa familiar, y sobre su reciente ingreso de tiempo completo. Al respecto, trabajos previos, en secuencia con la presente investigación, estudiaron el proceso de formación y la etapa de vinculación de descendientes (Lozano, 2008; Lozano y Urbano, 2008). Se hace relevante, por consiguiente, que este trabajo tenga como foco central el compromiso y satisfacción de descendientes con su primer cargo de tiempo completo en la empresa familiar, lo que constituye la primera etapa de pertenencia de los descendientes a la empresa.
En la formación de descendientes se han hecho varias consideraciones. Por un lado, la formación en valores se considera un tema de gran importancia en la etapa de formación en la niñez y juventud, antes de la entrada de tiempo completo por parte de los potenciales sucesores (Wortman, 1994; Lozano, 2003; García y López, 2001 y 2003; García, 2001; Drennan et al., 2005; Lambrech, 2005). Otro tema clave en formación es el desarrollo de habilidades de liderazgo en los potenciales sucesores (Turner, 1995; Ball y Beasley, 1998; Lozano, 2004) en el área de sucesión de las empresas familiares. Todo lo cual se complementa con un nivel de formación en áreas administrativas o en un área técnica importante, esto es, educación formal que le dé capacidades al potencial sucesor de trabajar en la empresa familiar (Goldberg, 1996; Morris et al., 1997; Howort y Assaraf, 2001; Venter et al., 2005; Lambrecht, 2005) si se vincula. Se atienden también las intenciones de descendientes a involucrarse y tomar participación en la empresa familiar (Birley, 1986 y 2002; Stavrou, 1999). Por otro lado, se han explorado los factores que influencian la decisión de tomar parte en la empresa (Dumas et al., 1995), como también los momentos y modos de entrada (Harvey y Evans, 1994; Lozano y Urbano, 2008).

En complemento, la literatura sobre sucesión, más enfocada a nuestro interés que es la satisfacción y compromiso con el primer cargo a tiempo completo, evidencia algunos énfasis que se exponen a continuación y que, junto con los conceptos de la teoría de recursos y capacidades, expuesta más adelante en el 
apartado 2.2, orientaron hacia los temas centrales de la investigación.

Lambrecht (2005) expone un proceso de sucesión de varios pasos. En cumplimiento del objetivo de esta investigación, interesa saber que el quinto paso ocurre con el inicio oficial del potencial sucesor en la empresa, a partir de lo cual debe pasar por varios departamentos de la misma e irla descubriendo más en detalle, así como a su sector y a los clientes.

En esta etapa el predecesor o las personas encargadas deben dar el apoyo necesario para que el potencial sucesor aprenda de sus errores y asuma tendencia a la innovación. Sin embargo, este importante paso en la vida profesional del potencial sucesor, requerirá de un sentimiento de satisfacción y compromiso con su trabajo al entrar a la empresa familiar, pues de ello dependerá en buena medida su desempeño futuro.

Sharma et al. (2001) desarrollan un modelo sobre satisfacción con el proceso de sucesión en empresas familiares bajo las premisas de la teoría de Stakeholder, así como varios aspectos de teorías de la organización, comportamiento y economía. En el modelo se propone que la satisfacción con el proceso de sucesión está directamente afectada por a) propensión del predecesor de ir cediendo el espacio, b) la voluntad del sucesor a tomar las riendas, c) acuerdo familiar para mantener la familia involucrada en la empresa, d) aceptación de roles individuales, e) plan de sucesión. Estudio éste que continúa con su trabajo sobre predictores de satisfacción con el proceso de sucesión en empresas familiares (Sharma et al., 2003a) en el cual se enfatiza que la sucesión exitosa tiene dos dimensiones en las empresas familiares: la satisfacción con el proceso de sucesión y el desempeño de la empresa después de la sucesión; aunque el estudio se concentra en el primero.

Por otro lado, Chrisman et al. (1998) y Sharma y Rao (2000) encuentran que la integridad y el compromiso con la empresa son los atributos de desempeño más importantes del sucesor, lo que hace pensar en la necesidad de enfatizar tempranamente el desarrollo de estos valores.

En su trabajo sobre desarrollo de carrera de adolescentes mayores Eckrich y Loughead (1996) encuentran que a medida que los descendientes de empresas familiares se vuelven más conflictivos con sus padres, es menos probable que se comprometan con la opción de carrera dentro de la empresa. Se infiere, que si encuentran un ambiente de baja armonía al vincularse, el compromiso y la satisfacción con el primer cargo serán bajos o quizá nulos.

En el plano del compromiso es preciso destacar los avances de Lansberg y Astrachan (1994) quienes para encontrar la influencia de las relaciones familiares en el proceso de sucesión realizaron un estudio en el cual sus variables independientes se basan en conceptos como la cohesión familiar y la adaptación familiar. Para ellos la relación familiar juega un papel muy importante en la planeación de un sucesor, lo cual está medido por dos componentes: a) Compromiso familiar con la empresa. Cuando el propietario involucra a la familia en la empresa, ven la firma como una extensión de sus valores, identidad, cultura y tradiciones, y siempre intentan asegurar la vitalidad y longevi- 
dad de la empresa. b) Calidad de la relación entre el dueño-administrador y el sucesor. Una buena relación entre estos dos presenta como características la confianza, ayuda mutua, buena comunicación, deseo de adquirir conocimiento, entre otras.

Dentro de sus conclusiones señalan que el compromiso familiar a la empresa es un gran medidor que influencia la cohesión familiar sobre la planificación de la sucesión y el entrenamiento del sucesor.

Las investigaciones que se han dedicado a señalar los atributos deseables en los posibles sucesores de la empresa familiar, han observado que es crucial en estos individuos la existencia del compromiso (Sharma e Irving, 2005). Miembros familiares comprometidos están más propensos a seguir una carrera dentro de la empresa familiar, a ser cooperadores en la transición de la toma de liderazgo, y a estar satisfechos con el proceso de sucesión general. Razón por la cual aconsejan promover el compromiso con su empresa entre la siguiente generación de posibles sucesores.

El compromiso se define como una fuerza que se experimenta como marco mental o estado psicológico que induce al individuo hacia el curso de una acción relevante para uno o más objetivos. Sharma e Irving (2005) se enfocan en la búsqueda del entendimiento de las actitudes, entre la siguiente generación de sucesores de propietarios de empresas familiares, para seguir una carrera dentro de la firma. Indican, además, que las razones de haber ingresado a la empresa familiar pueden variar y que estas razones se ven reflejadas en cuatro diferentes tipos de compromiso que moldean el comportamiento de los sucesores: compromiso afectivo, compromiso normativo, compromiso calculador, compromiso imperativo.

Un individuo con alto compromiso afectivo hacia la organización demuestra gran credibilidad, aceptación y emoción hacia las metas de la empresa, de la misma manera que gran deseo por contribuir a alcanzarlas y, de esta forma, alineándolas con sus propias metas. Al existir esta alineación, se funda una creencia de que las aspiraciones profesionales pueden ser satisfechas con el contexto de la empresa.

Un individuo con alto compromiso normativo hacia la firma tendrá como factor pujante una obligación en lugar de un deseo intrínseco para seguir en ella, pero a pesar de esto no lo ve como un factor negativo. Sin embargo el sentido de obligación puede ocasionar dificultad en la creación de buenas relaciones dentro de la empresa.

Un individuo con alto grado de compromiso calculador siente que es su deber trabajar en la empresa familiar porque de otro modo saldría perdiendo por la inversión hecha allí previamente.

Un individuo con alto grado de compromiso imperativo percibe que en su vida hay ausencia de alternativas de carrera. Este tipo de compromiso está basado en el sentimiento de desconfianza e incertidumbre acerca de la habilidad de que el individuo pueda seguir una carrera exitosa fuera de la empresa familiar.

Como Meyer y Hercovitch (2001) manifiestan que es deseable promover el compromiso afectivo, en esta investigación se ha acogido este concepto, pues el 
uso típico del término compromiso en la literatura de las empresas familiares es consistente con la definición de compromiso afectivo (Sharma e Irving, 2005).

Meyer y Herscovitch (2001) dicen que el compromiso debe tener una "esencia del núcleo" a pesar del contexto en el que es estudiado y que, por lo tanto, debería ser posible desarrollar un modelo general de compromiso del lugar de trabajo, por lo cual propone uno basado en que el compromiso: a) es una fuerza que vincula a un individuo a un curso de acción relevante para alcanzar metas, b) puede estar acompañado por diferentes criterios que juegan un papel en la determinación del comportamiento.

En complemento a ello, Sharma et al. (2003b) indican que el compromiso se refiere a la extensión a la cual los miembros de la familia desean la perpetuación de la empresa en manos de la familia. Es una expresión de su aprobación de la sucesión. Por tanto un alto compromiso familiar debería fortificar la intención del predecesor de continuar la sucesión. Es más, esa intención debería ser comunicada a los demás miembros de la familia, pues de esta manera la propensión a vincularse podría ser incrementada, por simple asunción de que habrán más espacios para obrar con libertad y exponer las capacidades sin mayores limitaciones.

Así, yéndonos ahora al plano de la satisfacción, si un descendiente toma un primer cargo y no se le da autonomía y libertad en él, es decir, si se dejan las cosas en manos de otro, influencia directamente su satisfacción con las funciones encomendadas.

Sharma et al. (2001) han estudiado los factores que influencian la satisfac- ción inicial con los procesos de sucesión en las firmas familiares. Sus desarrollos teóricos sugieren que la satisfacción con el proceso de sucesión en las firmas familiares es realzado con la propensión del titular de hacerse a un lado, la buena voluntad del sucesor para hacerse cargo, acuerdo entre miembros familiares para mantener la implicación familiar en el negocio, aceptación de los papeles individuales, y la planeación de la sucesión.

En complemento, en su estudio sobre predictores de satisfacción con el proceso de sucesión Sharma et al. (2003a) indican que el proceso de sucesión tiene dos dimensiones interactivas: la satisfacción con el proceso y la efectividad del mismo. El primero es un análisis subjetivo del proceso y de la decisión. El segundo es una determinación objetiva del impacto de la decisión en el rendimiento de la firma.

Entender estos factores que influencian la satisfacción con el proceso de sucesión, es un relevante tópico de estudio. Ello tiene, sin embargo varios rasgos particulares, razón por la cual en este trabajo se hace énfasis en la primera fase de vinculación del descendiente, porque la insatisfacción con el primer cargo a tiempo completo puede influenciar de manera adversa la relación intrafamiliar y entorpecer la continuidad del potencial sucesor, es decir, su carrera en la empresa familiar. Pero aún si el potencial sucesor continúa a pesar de su insatisfacción con el primer cargo, esto puede causar conflictos futuros en su trabajo con la empresa, y poner en riesgo la continuidad de la misma.

Estos conflictos generalmente están asociados a un deterioro de la armo- 
nía en el sistema empresa/familia, en el que mantener las buenas relaciones es extremadamente importante (Golberg, 1996; Sharma et al, 2001; Lozano, 2006). Al respecto, en su estudio sobre porqué algunos sucesores son capaces de obtener utilidades para sus empresas y otros no, Golbert (1996) encuentra que los sucesores efectivos tuvieron una relación con los padres significativamente buena, tuvieron una niñez más alegre, fueron introducidos a la empresa a una edad temprana y empezaron a trabajar tiempo completo a una edad también temprana.

Las encuestas muestran que todos los sucesores efectivos se han visto beneficiados por las múltiples relaciones con sus mentores. Los sucesores menos efectivos aparentemente tuvieron otras alternativas antes de integrarse a la empresa y, por lo tanto, puede ser una de las razones por las cuales hay falta de confianza acerca de su futuro, pues sienten que podrían estar perdiendo otras oportunidades valiosas.

Por otro lado, los consejeros de empresas familiares pueden alertar a los líderes de estas empresas sobre el posible riesgo de ejercer influencia para presionar a los hijos en su primer trabajo de tiempo completo en la firma. El padre puede tal vez hacerlos unir a la compañía, pero el poco compromiso con la opción puede producir un empleado poco entusiasta y falto de realizaciones. En consecuencia, sin la disposición del potencial sucesor en el primer cargo, el desempeño tendrá que procederse con su disgusto y resistencia. Pero una asunción presionada de la toma del primer cargo, afectaría negativamente la satisfacción. En contraste, cuando las familias o el an- tecesor deciden retener el liderazgo de la firma dentro de la familia sin presiones, el potencial sucesor que sí quiere la tarea adquiere legitimidad y poder, influenciando de manera positiva todo el proceso. EI potencial sucesor se siente acogido y esto influye positivamente en la satisfacción con el primer cargo. Lo cual, en consecuencia, se complementará con el deseo de hacer las cosas bien y llegar a objetivos importantes para la empresa, es decir, tener compromiso con el trabajo.

\section{Teoría de recursos y capacidades de la empresa}

La teoría de recursos y capacidades emerge como una teoría de la ventaja competitiva mediante Wernelfelt (1984), que acoge la heterogeneidad de la empresa, con aportes posteriores de autores de la teoría de la economía de la organización y de la dirección estratégica. Algunos autores (Diericx y Cool, 1989; Barney, 1991; Peteraf, 1993) hicieron contribuciones a la teoría de recursos y capacidades, derivando estas en la empresa como unidad de análisis.

Dado que la teoría de recursos y capacidades tiene como propósito principal identificar el potencial de la empresa para establecer ventajas competitivas mediante la identificación y valoración de los recursos y capacidades que posee o los que puede acceder (Navas y Guerras, 1998), es comprensible que la ventaja competitiva sea vista como el producto de implementar estrategias de creación de valor que no son simultáneamente implementadas por otras empresas. Estas ventajas competitivas son consecuencia de los recursos claves que tiene la empresa. 
La teoría establece, por tanto, que el que algunas empresas consigan resultados superiores radica en que ellas disponen y controlan recursos críticos y capacidades especiales que las diferencian de las demás. Ahora, Cuando los beneficios generados por estas estrategias no pueden ser replicados por la competencia, esta ventaja competitiva se convierte en ventaja competitiva sostenible.

El análisis de los recursos y capacidades deriva estratégicamente en tres aspectos claves (Grant, 1996a y 1996b): 1) definir la estrategia que explote los principales recursos y capacidades; 2) garantizar que los recursos y capacidades se empleen bien y sean totalmente explotados; 3 ) construir la base futura de recursos y capacidades cubriendo las carencias actuales, mejorando lo existente e invirtiendo en el desarrollo de nuevos recursos que puedan ser valiosos para mejorar competitividad y plantear nuevas estrategias.

Este último aspecto plantea invertir para lograr un desarrollo interno de recursos y capacidades. De no ser posible el desarrollo interno, la empresa debe estudiar otras alternativas, entre ellas la adquisición directa en el mercado (Navas y Guerras, 1998). De aquí se desprende que estos recursos y capacidades también es factible detectarlos en los hijos de propietarios, teniendo como fuente la familia; a los cuales se puede desarrollar a alto nivel de una manera integral en diversos papeles empresariales. La familia como fuente de recursos y capacidades provee capital, personas, conocimiento, tiempo, ideas, fuerza moral, entre otros. Los hijos hacen parte de este set de recursos y capacidades que la familia pue- de proveer a la empresa, pero deberán exponerse a los procesos de selección que estén definidos en la organización.

Cuando el potencial sucesor, en su edad infantil o adolescente, se involucra progresivamente con las rutinas de la empresa, va asimilando, también progresivamente, conocimiento expreso y conocimiento tácito, con frecuencia, de la mano del predecesor. Aunque se suele encontrar regularmente la participación de trabajadores, directores, y mentores (Cabrera et al., 2001). La diferencia entre un hijo que se vincula a la empresa de la familia, y otra persona no familiar que también lo hace, consiste en que el hijo, aún sin proponérselo él ni sus padres, posiblemente ha hecho un apropiamiento de la actividad empresarial de estos antes del momento de entrar a la empresa. En tanto el segundo, el no familiar, tiene menos posibilidades de haberlo hecho. El apropiamiento del hijo puede estar en términos de valores, conocimientos de negocios de la familia, contactos con clientes de la empresa, habilidades empresariales y, por lo tanto, más posibilidades de entender el contexto de negocios de la familia (Lozano, 2008).

Siendo esto así, desde la perspectiva de recursos y capacidades se estarían justificando los esfuerzos que los propietarios hagan para vincular a los hijos de manera formal y con dedicación de tiempo completo en la empresa. La perspectiva de recursos y capacidades nos estaría explicando porqué preparar a los descendientes para que hagan parte del negocio familiar, y también porqué deberían asumir con un importante nivel de satisfacción y compromiso su primer cargo de tiempo completo. La perspectiva de 
recursos y capacidades explicaría que la entrada formal de los hijos a la empresa se estaría dando porque reúnen unas condiciones que favorecen la búsqueda de rentas para ella.

\section{Primera fase de pertenencia a la empresa y proposiciones}

Desde la literatura y el estudio de casos, dos grandes factores conforman los pilares de la estructura de la primera etapa de pertenencia a la empresa. Consecuentemente, de la literatura y del proceso iterativo de revisión y análisis de la información de los casos, se han reconfirmado estos dos factores, satisfacción y compromiso, y se han derivado los ítems que conforman sus respectivos constructos, tal como se expone en el Cuadro 1.

Aunque en la literatura se hace mención a la satisfacción y al compromi- so, estos no son asociados directamente al primer trabajo de tiempo completo del descendiente en la empresa familiar. En realidad estos dos factores definen la pertenencia del descendiente en la primera fase de vinculación a tiempo completo. Lambrech (2005) ha abordado el concepto pertenencia al derivar un modelo explicativo que muestra el principio de gobierno en el cual la continuidad de la empresa familiar está basada consiste en que el individuo pertenece a la familia, la que pertenece a la empresa. Es decir, el individuo pertenece a la empresa.

Si bien es cierto que en el caso de los descendientes la pertenencia se va dando gradualmente, su verdadera expresión conceptual se daría cuando el descendiente esté vinculado a tiempo completo. Pero, además de ello, dos condiciones deben estar presentes: el sentimiento de gusto por las tareas encomendadas $\mathrm{y}$, adicionalmente, que estas ta-

\section{Cuadro 1 \\ Estructura de la etapa de Pertenencia}

\begin{tabular}{ll}
\hline & \multicolumn{1}{c}{ Ítems } \\
\hline & Procuró tener buenas relaciones con su jefe y los compañeros de trabajo \\
& Aplicó sus conocimientos en la empresa \\
& Se interesó por aprender del cargo y de otros que lo complementaban \\
Factor & Experimentó sentimientos de gusto por lo que realizaba \\
Hizo esfuerzos por desarrollarse profesionalmente \\
Ejerció comportamientos de liderazgo \\
Sintió confianza al realizar lo encomendado
\end{tabular}

Mantuvo sentimientos de pertenencia a la empresa

Factor

Participó voluntariamente en el proceso de toma de decisiones

COMPROMISO Procuró dar cumplimiento profesional a las metas establecidas

Se esmeró por los intereses empresariales

Fuente: Elaboración propia. 
reas efectivamente se ejecuten y se hagan bien. En otras palabras, estas dos condiciones hacen referencia a los factores Satisfacción y Compromiso.

Conforme a todo lo anterior, literatura y estudio de casos, esta investigación nos ha llevado a identificar importantes proposiciones que se muestran a continuación. Es importante aclarar que las citas específicas de los entrevistados, que refuerzan lo observado, tienen al final un distintivo $\mathrm{Pn}$, donde $\mathrm{P}$ significa persona entrevistada y $n$ indica el orden en que aparece la entrevista en la unidad hermenéutica del ATLAS/ti. Enseguida del distintivo $\mathrm{Pn}$ aparece el número del párrafo donde se ubica la cita en el texto de entrevista.

\section{Satisfacción}

\section{La Satisfacción se refiere al nivel} de agrado con las experiencias que se viven durante el desempeño del primer cargo.

En la literatura sobre empresas familiares la confianza en las capacidades de los descendientes ha sido resaltada (Venter et al., 2005). La valoración y confianza en las capacidades de una persona origina satisfacción en ésta, pues significa recibir reconocimiento a algo que se posee y eso genera agrado. Entre miembros familiares la confianza refleja la voluntad de llenar expectativas, apoyarse el uno al otro, intercambiar confidencias, operar dentro de sistemas con equidad (Lumpkin et al., 2008: 133). Es entendible entonces que la confianza depositada en las capacidades del descendiente en su primer cargo le hará sentir satisfacción al desempeñarlo. Más aún por el hecho de que esto lo va legitimando ante familiares y no familiares (De Massis et al., 2008:188). Veamos los siguientes enunciados emitidos por los entrevistados.

"Pude demostrar afuera donde en cierto modo me van a valorar por lo que soy y no que en cierto modo les toque aguantarme porque soy la dueña o la hija del dueño, sino poder demostrar la capacidad profesional en otra parte, entonces en ese sentido fue muy importante para mí". P3 (115:115).

"Cuando ya tenía máquinas bonitas y grandes era mucho más agradable para mí, porque ya me sentía mucho más importante, ahí ya tenía salario pero mi responsabilidad seguía siendo muy fuerte". P4 (39:39).

"Sentir que lo que estaba aprendiendo era práctico, o sea, poder sentirme que yo estaba haciendo algo que las demás personas lo valoraban, que mi trabajo llegara a la compañía y la demás gente dijera, esto era lo que yo necesitaba, o que inmediatamente yo entregaba el trabajo lo ponían en práctica". P4 (88:88).

"Me he sentido muy halagada cuando Alfredo me presentó a Mauricio, le dijo, mira la importación que hemos hecho...". P9 (143:143).

"Siempre me dije a mí mismo, yo aquí tengo que demostrar que sirvo, que no quiero ser el culpable ni darle la razón a la mayoría de los libros en cuanto que las empresas familiares se terminan en la segunda o tercera generación". P11 (60.60).

Proposición 1: Los descendientes de la empresa familiar serán más propensos a sentir satisfacción con las activida- 
des asignadas en su primer cargo, cuando sienten que los integrantes de la familia confian en sus capacidades.

Se tiene también que la armonía familiar es un tema que aparece con frecuencia en las investigaciones sobre empresas familiares (Dun, 1999; Venter, 2005; Lozano, 2006; Björnberg y Nicholson, 2007). Recientemente De Massis et al. (2008:188) resaltan los factores de relación entre grupos o individuos, profundamente asociados el proceso de sucesión. Observar y confirmar que los miembros de la familia tienen un buen nivel de entendimiento y comunicación producirá buen ánimo en los descendientes en el desempeño de su primer trabajo de tiempo completo. Las expresiones de los descendientes así lo hacen notar.

"Era un trabajo agradable, era muy familiar, en ese momento no éramos tantos. No familiar en el sentido de que había mucha gente de la familia, sino por la relación con la gente, había gente que llevaba varios años con nosotros". P3 (139:139).

"Siempre hemos trabajado bien, lo que ha facilitado es que cada uno tiene como su área, no somos competencia en cuanto que digamos yo me meto en tu área, tú te metes en la mía, porque cada uno tiene su especialidad y cada uno ha respondido por el área que maneja. Entonces eso en cierto modo ha facilitado el funcionamiento, y yo creo que no ha habido grandes crisis y grandes dificultades". P3 (223:223).

"Hemos hecho, diría yo, que muy buen equipo y hemos trabajado sabroso". P3 (235:235).

"Estando con los mecánicos era muy rico porque yo era muy amigo de ellos, yo jugaba fútbol, yo jugaba voleibol, y habían canchas en la fábrica y entonces yo tenía horario de obrero común y corriente, y equipo de seguridad común y corriente. Yo pasaba muy sabroso". P4 (39:39).

"Uno nunca ha sido distante, aquí hay otra cosa que pasa y es que vos estás con el que barre, con el que manda, con el que... a todo nivel. Nunca hay barreras ni algo así, ni yo soy la dueña y ustedes son los que trabajan, no". P5 (143:143).

"Ellos se motivaron, tal vez por el modo de ser mío, por que yo siempre me sentía contento con lo que hacía, yo creo que eso también los motivó mucho a ellos". P6 (79:79).

"El ambiente muy bueno y con todo el mundo y no solamente de esa época sino de ahora también", P8 (79:79).

"Cuando empecé los primeros días y ahora, el ambiente, el ambiente que se siente aquí, es tan familiar, es un ambiente cálido, bueno". P9 (147:147).

"Siempre he admirado mucho eso, como esa parte, esa integración, esa unión familiar, que cada uno respeta al otro como persona. Las decisiones que se toman son digamos de común acuerdo, siempre hay una armonía muy interesante que es lo que ha logrado que la empresa haya perdurado en el tiempo". P11 (78:78).

"Muy bien, parecemos unos amigos, no solamente con Mauricio no, sino con mis otros hijos también, es maravilloso, la relación papá-hijos, ha sido maravillosa, siempre...respetándole sus ideas, su forma de pensar, su forma de obrar". P12 (115:115). 
Proposición 2: Los descendientes de la empresa familiar serán más proclives a sentir satisfacción con las actividades asumidas en su primer cargo, cuando las relaciones que perciben entre los familiares son de diálogo y armonía.

Chirico y Salvato (2008) han resaltado la integración de conocimiento especializado entre familiares como esencial para tener éxito en mercados dinámicos. En una familia propietaria la preparación de todos sus miembros es bien aprovechada si sus conocimientos son combinados para su aplicación en la empresa. El miembro familiar recién vinculado de tiempo completo se verá favorecido de ello para la aplicación de sus conocimientos en la empresa, lo cual seguramente hará con agrado. Apreciemos los comentarios de los entrevistados.

"Yo recuerdo que cuando estaba en el conmutador estaba como empezando la carrera apenas, digamos como haciendo cosas y estando en contacto con la empresay con la gente". P3 (71:71). "El hacer trabajos en la Universidad que realmente servían dentro de la empresa eso disparó la motivación impresionante, entonces cada vez los proyectos fueron más grandecitos, más grandecitos, y eso fue lo que más me motivó". P4 $(40: 40)$.

"Yo aprendí a hacer baterías con la gente en planta, aprendí a hacer baterías viajando, aprendí a hacer baterías estudiando, revolcando libros". P4 (68:68). "Lo interesante es que es ahí donde empiezas a complementarte con la carrera, porque lo que estás viendo en la universidad no se te está quedando teóricamente, entonces lo asimilas y empiezas a aplicar cosas". P11 (60:60).

\section{Proposición 3: Los descendientes} de la empresa familiar son más propensos a sentir satisfacción con las actividades asignadas en su primer cargo, cuando sienten que el conocimiento académico adquirido es aplicable, al menos parcialmente, a las distintas actividades de la empresa.

Una búsqueda permanente de realización del ser humano es poder trabajar en lo que le gusta, lo cual se da también en el ámbito de las empresas familiares. De Massis et al. (2008) argumenta que si las tareas encomendadas al potencial sucesor no son de su agrado, su motivación decrece y no se sentirá satisfecho, originando la probabilidad de dejar la empresa; caso contrario ocurrirá si las actividades a realizar son de su agrado e interés. De otro lado, Venter et al. (2005) encuentra gran importancia en las necesidades personales de alineamiento del potencial sucesor con el trabajo en la empresa familiar. Alineación que debe ser clara desde el inicio del primer trabajo de tiempo completo en la empresa. A continuación algunos enunciados de los descendientes vinculados.

"Yo me vinculé con Ganales fue a través de los negocios de servicios, a mí me gusta el comercio y la venta de servicios". P2 (99:99).

"Era muy chévere para mí empezar a dirigir montajes mecánicos en la fábrica, ese fue prácticamente mi primer trabajo". P4 (39:39).

"Soy un apasionado de lo que hago, para mí es mucho más que querer lo que hago, yo soy realmente apasionado, para mies pasión y esa pasión me genera problemas, porque no tengo horarios, no tengo restricciones, entonces descuido mi familia y lo personal". P4 (133:133). 
"Te vas a encargar de la infraestructura de la empresa, entonces yo dije ahhh me tocó lo mío, o sea, se dieron las cosas, fue re-hacer todo lo que había en toda la compañía, en ese momento eran 3 compañías, eran más de 70 puntos de venta en todo el país". P5 (91:91). "Mi padre había cambiado su destino de ganadero a cañicultor y eso no me llamaba la atención. Ir a ver todos los días las matas de caña que en esa época había poquito pero... eh... preferí las bombas por que me daba más visión de poder estar viajando, saliendo, conociendo gente". P7 (166:166).

"El trabajo me gustaba, a mí me gustaba muchísimo el trabajo porque era lo que yo buscaba". P8 (43:43).

"Comencé muy contenta, la gente me decía, uy! que pereza uno estar cobrando, en la labor de chepita, me parece hartísimo, y yo decía, qué tiene de malo, a mí me parece riquísimo, estar hablando por teléfono haciendo este tipo de actividad". P9 (167:167).

"Nunca he pensado en otro trabajo o en decir voy a buscar otra experiencia en otra empresa...no, yo creo que aquí ya me quedé pero porque quiero quedarme". P11 (74:74).

Proposición 4: Los descendientes de la empresa familiar estarán más inclinados a sentir satisfacción con las actividades asignadas en su primer cargo cuando sientan agrado por lo que realizan y vivencian en ellas.

\section{Compromiso}

El Compromiso se refiere a la fuerza del descendiente que lo induce a acciones relevantes para alcanzar

\section{objetivos de beneficio para la empresa familiar.}

Los tempranos contactos del potencial sucesor con la empresa para establecer relaciones con los grupos de interés le permite entender la cultura y las cosas de la empresa que no son tan conocidas (De Masiss et al., 2008) y de esta manera ir identificándose con la empresa, ir sintiendo que pertenece a ella. Se ha encontrado que esta es una razón por la que la familia prefiere que la firma sea continuada por miembros familiares (Lambrech, 2005). El descendiente expondrá compromiso en su primer cargo a tiempo completo en tanto el sentimiento de encuentro y pertenencia a la empresa sea evidente. A continuación se exponen algunos enunciados emitidos al respecto por los entrevistados.

"No ninguna, yo no puse ninguna condición, es más, trabajé 6 meses sin sueldo, tenía un reto que era un tema de que el hotel no se cerrara, en una zona agrícola donde no hay sino visitadores agrícolas y de casas, y bueno, crear turismo". P2 (103:103).

"Yo la empresa la manejo como si fuera mía, nunca pienso que si quiera existe nadie más, mis ideas, mi cerebro y mi todo está dispuesto para eso". P2 (143:143).

"No me veo haciendo otra cosa, es más el cariño que uno le tiene a la empresa que otra cosa". P4 (84:84).

"El ahorro nuestro está generado en patrimonio empresarial, y nosotros dedicamos todas las horas hábiles posibles a que ese patrimonio crezca". P4 (100:100).

"Es un sentido más sentimental el que tiene uno por toda la historia que tiene la 
empresa, por lo que significa, que una motivación salarial". P11 (66:66).

"Es muy distinto de pronto cuando uno es un empleado a ser parte de una empresa familiar, cuando uno quiere la empresa, digamos la palabra que me gusta utilizar es que vale la pena dar el todo por la empresa, ahí no hay límites, no hay horarios, no hay nada, lo que haya que hacer" P11 (86:86).

"Realmente me llama mucho la atención que Mauricio quiera tanto la empresa, es decir, él considera que la empresa es algo supremamente importante y le tiene un amor tremendo". P12 (99:99).

Proposición 5: Los descendientes de la empresa familiar serán más proclives a comprometerse con las actividades asumidas en su primer cargo, cuando tengan un sentimiento de identificación y pertenencia con la empresa.

El tema de liderazgo ha sido y continúa siendo uno de los más reconocidos en el ámbito organizacional (Sorenson, 2000; Abell, 2006). En concordancia con ello se ha expuesto que la habilidad de desarrollar liderazgo en la generación sucesora es crucial para la supervivencia de la empresa familiar (Turner, 1995) y lograr que esta futura generación maneje de forma exitosa los problemas que surgen a la hora de manejar la empresa. Por ello alienta con una propuesta para desarrollar liderazgo con bastante anticipación, dentro de lo cual se incluye la toma de decisiones. De ello se deduce la importancia de las oportunidades para la toma de decisiones durante el desempeño del primer cargo a tiempo completo en la empresa, ello repercutirá en su nivel de compromiso con lo que desempeña. Algunas expresiones de los descendientes se exponen a continuación.

"Las decisiones más importantes eran montar la fábrica de vinos, montar la procesadora de frutas, comprar nuevas fincas nuevas tierras". P1 (219:219).

"Tomo decisiones en el hotel y desde hace ya dos años he estado en la parte agroindustrial, hace ya año y medio comencé a participar en las reuniones de junta directiva, entonces asistía, miraba". P2 (107:107).

"El cargo no era un cargo de grandes decisiones, era un cargo llamémoslo más bien de soporte, o sea que ahí no había lugar a decidir cosas de gran trascendencia". P3 (207:207).

"Eso fue construido por años, o sea, nosotros no vinimos a tener decisión propia o capacidad de decisión sino hasta como 6 ó 7 años, de estar vinculados, antes éramos simplemente un empleado que hacía tareas como muchos". P4 (96:96).

"Mis hermanos y todo si siempre me han metido en todo, digámoslo, así sea a nivel de escucha; pero al principio yo no tenía ni idea de nada, o sea, yo iba a la Junta a todo, pero uno al principio no sabía si 10 era bueno y 20 era pésimo, no, ni idea". P5 (119:119).

"La palabra del jefe siempre es la palabra del jefe y finalmente se hace lo que él diga, pero casi la decisión era totalmente de uno, de qué hacer; consultas, se pide aprobación y todo pero generalmente el que está al pié del cañón es el que sabe lo que hay que hacer". P8 (67:67). 
Proposición 6: Los descendientes de la empresa familiar estarán más inclinados a comprometerse con las actividades asignadas en su primer cargo, cuando se les otorgue espacios para tomardecisiones al menos a nivel de la función jerárquica que desempeñen.

Quien entre a trabajar a una organización que se está desarrollando, o al menos tiene potencial de desarrollo, de inmediato hay un compromiso con el desarrollo de esa empresa. De Massis et al. (2008), declara que la expectativa de un crecimiento en el mercado o en las finanzas, aumenta el atractivo del potencial sucesor por la empresa familiar. Incluso, sugiere que hay una correlación positiva entre el tamaño de la empresa y las intenciones de vinculación de los descendientes, y una vez dentro se asume un compromiso a la espera de seguir siendo próspero. El desarrollo y crecimiento de las empresas familiares (Upton et al., 2001; Ward, 1997; Stafford et al., 1999; Moreno y Casillas, 2008) además de ser atractivo, crea sentimientos de responsabilidad y apoyo que redundarán en bien de todos. Las siguientes son algunas de las manifestaciones de los encuestados.

"Todos luchamos en la empresa de manera que la empresa progrese bajando costos...bajando costos, porque la competencia hoy en día es tan fuerte que ya los clientes llegaron a un punto que no se puede trabajar a punta de aumentar los precios sino a punta de bajar los costos". P6 (168:168).

"...no, al contrario, ellos como que pedían más cuerda, ellos siempre han pensado y han sentido que la empresa tiene que desarrollarse y ellos son muy activos en ese sentido". P6 (176:176).
“... buscando nuevos retos nuevas metas buscando nuevos objetivos buscando nuevos clientes...". P7 (198:198).

"Siempre he tenido la mentalidad de que si hay que hacerlo se le hace, siempre con la visión de decir hoy voy a hacer esto, pero yo se que va a ser por algo, que más adelante uno va a cosechar todas esas semillas que sembró". P11 (70:70).

Proposición 7: Los descendientes de la empresa familiar estarán más inclinados a comprometerse con las actividades asignadas en su primer cargo, cuando perciban que al trabajar por la empresa existen posibilidades para que la organización se desarrolle y crezca.

Moreno y Casillas (2008) resaltan que las empresas en crecimiento son las que generan más trabajos, lo cual se hace relevante para la sociedad. Otros autores dan gran importancia a las acciones de tipo social que se generan desde la empresa y en asocio con otros actores con los cuales se interactúa (Bartholomew y Smith, 2006; Barnett y Kellermanns, 2006). Estos aportes sociales tienen gran relevancia para los potenciales sucesores de empresas familiares quienes, al vincularse, encuentran en ello un elemento que les produce más compromiso con la empresa. Examinemos algunas de las expresiones de los entrevistados.

“... a 220 personas que trabajaban en la empresa, sí, a los más antiguos entonces me preocupé por vivienda, salud, educación y recreación; se hizo un parque recreativo con piscina para la gente.... P1 (223:223).

"Cuando una de las compañías está en peligro o se piensa cerrarla, porque he- 
mos cerrado varias; así como se abren dos o tres al año se cierran otras también; reflexiono sobre qué va a pasar con las personas que están ahí dentro, entonces generalmente intervengo a profundidad para salvarlas". P2 (115:115)

"Me siento muy satisfecha porque digamos me gusta la región donde están las compañías, pienso siempre que allí hay progreso y prosperidad gracias a las compañías del grupo. Me siento profesionalmente desarrollada". P2 (119:119).

"Me siento muy bien con que ellos manejen la organización y que la hagan crecer para bien de todos, no sólo de la familia, sino para la comunidad en que vivimos, para el país, para el progreso en general". P6 (292:292).

Proposición 8: Los descendientes de la empresa familiar serán más propensos a comprometerse con las actividades asignadas en su primer cargo, cuando sientan que desde la empresa se pueden o se podrán hacer aportes sociales a la comunidad interna o externa de la misma.

Alta relevancia se le ha dado también al tema de la propiedad en empresas familiares (Williams, 1992; Ward y Dolan, 1998; Habbershon y Pistrui, 2002). El poder de la propiedad tiene una clara relación con el proceso de sucesión. Al respecto De Massis et al. (2008) indica que la posibilidad de perder control accionarial o la ausencia de suficientes atractivos para inversiones adicionales, puede frustrar que la sucesión sea hecha con recurso humano de la familia propietaria. En concordancia con esto, al tratar el involucramiento en la empresa familiar, Chris- man et al. (1998) aseveran que un sucesor puede ser involucrado en la empresa familiar a través de acciones, lo que implicaría más inclinación hacia un cumplimiento responsable de las actividades, incluso desde el primer trabajo de tiempo completo. Es interesante examinar las sentencias de los entrevistados.

"Gracias a Dios nunca me vi empleado de nadie, entonces al principio yo trabajaba para esas empresas y hacía mas empresas, eso es algo que yo nunca paré". P1 (191:191).

"... la oportunidad de quedarme con una participación del Hotel, a mí la compañía que más me llamaba la atención era el Hotel". P2 (111:111).

"...yo diría que la opción de trabajar aquí, de trabajar en lo de uno". P3 $(119: 119)$.

"La sola motivación de poder desarrollarte como persona en algo que te gusta y que además de que te gusta es tuyo, no tiene comparativo con nada". P4 (120:120).

"Como ellos han visto que dentro de la empresa hay la oportunidad de desarrollar los conocimientos y que es la empresa de ellos, yo creo que han calculado ya, han aceptado que es mejor trabajar en la compañía que hacer cosas por aparte". P6 (248:248).

"Yo sabía que yo tenía una mejor oportunidad de hacer carrera trabajando aquí en Colpozos, que trabajando en cualquier otra parte". P8 (55:55).

"...yo sentía, pues, que en parte eso era un legado". P9 (187:187).

Proposición 9: Los descendientes de la empresa familiar estarán más propensos a comprometerse con las actividades asignadas en su primer cargo, 
cuando conocen que son poseedores o poseerán propiedad en ella.

\section{Conclusiones}

De las reflexiones planteadas a lo largo del artículo, pueden esgrimirse dos Conclusiones principales. La primera conclusión indica que la pertenencia a la empresa familiar en la primera fase de trabajo a tiempo completo, tiene sus dos cimientos en la satisfacción y el compromiso del descendiente con ese trabajo. Su trascendencia se extiende más allá del hecho de sentir gusto por el trabajo y de tener dedicación para hacerlo bien. Al parecer la pertenencia en esta primera fase de tiempo completo se tornaría explicativa de la propensión del potencial sucesor en hacer carrera en la empresa familiar, lo que debe probarse en estudios posteriores. La segunda conclusión se deriva del hecho de que la perspectiva de recursos y capacidades estaría justificando los esfuerzos que los propietarios hagan para vincular a los hijos de manera formal y con dedicación de tiempo completo en la empresa. La teoría explicaría que la entrada y posterior permanencia de los hijos en la empresa se estaría dando porque reúnen unas condiciones que favorecen la búsqueda de rentas para ella. Condiciones estas que también tendrán dependencia de la satisfacción y compromiso que se asuma por parte del potencial sucesor en el primer trabajo a tiempo completo, es decir, en su primera fase de pertenencia a la empresa. De esta manera, la preparación y el acompañamiento que se les brinde a los descendientes en esta primera fase de vincula- ción a tiempo completo, será clave en el desempeño y la continuidad de recursos valiosos para la empresa familiar.

Estas dos conclusiones se tornan interesantes para propietarios y descendientes de empresas familiares a la hora de definir las estrategias del proceso de sucesión, y también permite a los investigadores nuevos elementos para avanzar en el área. Por supuesto, una próxima investigación es el contraste de proposiciones; ya en forma de hipótesis, considerando una muestra representativa de empresas familiares colombianas.

Este trabajo permite avanzar en el estudio de las empresas familiares en cuanto que el inicio a tiempo completo en esta marca la verdadera pertenencia del potencial sucesor. Es decir, el concepto de pertenencia tiene su clave en el primer trabajo a tiempo completo en la empresa familiar. La pertenencia, por lo tanto, no hace referencia a experiencias de fin de semana o de vacaciones de verano; la pertenencia es un estado, fuerte o débil, de compromiso y satisfacción tácito o expreso entre la empresa familiar y el potencial sucesor. Este trabajo aporta en identificar los factores satisfacción y compromiso con sus respectivos componentes, lo cual se hace relevante en cuanto que las relaciones entre estos componentes conducen al planteamiento de proposiciones para darle continuidad al estudio. Con ello los empresarios y sus potenciales descendientes, podrán examinar esas relaciones y acordar comportamientos que hagan más efectivo el interaccionar entre padres e hijos en un entorno empresarial. 


\section{Referencias bibliográficas}

Abell, Derek F. (2006). "The Future of Strategy is Leadership". Journal of Business Research, 59, 310-314.

Astrachan, Joseph. H. y Shanker, Melissa. C. (2003). "Family business' contribution to the U.S. economy: Closer outlook". Family Business Review, 16(3), 211-219.

Ball, Rebeca W. y Beasley, Fred M. (1998). "Entrepreneurship awareness education: An example in an elementary school". Journal of Small Business Strategy, 9(1), 26-38.

Barnett, Tim y Kellermanns, Franz W. (2006). "Are We Family and are We Treated as Family? Nonfamily Employees' Perceptions of Justice in the Family Firm". Entrepreneurship, Theory and Practice. Noviembre, 837-854.

Barney, Jay. (1991). "Firm Resources and Sustained competitive Advantage". Journal of Management, 17(1), 99120.

Bartholomew, Susan y Smith, Anne D. (2006). "Improving Survey Response Rates from Chief Executive Officers in Small Firms: The Importance of Social Networks". Entrepreneurship, Theory and Practice, Junio, 83-96.

Birley, Sue. (2002). "Attitudes of OwnerManager's Children towards Family and Business Issues". Entrepreneurship, Theory and Practice, 26(3), 5-19.

Birley, Sue. (1986). "Succession in the Family Firm: The Inheritor's View". Journal of Small Business Management, 24(3), 36-42.

Björnberg, Asa y Nicholson, Nigel. (2007). "The Family Climate Scales - Development of a New Mesure for Use in Family Business Research". Family Business Review, 20(3), 229-246.
Cabrera, Katiuska; De Saá, Petra De; García, Desiderio. (2001). "The succession process from a resource and knowledge based view of the family firm". Family Business Review, 14(1), 37-46.

Cabrera, Katiuska. (2005). "Leadership transfer and the successor's development in the family business". The Leadership Quarterly. 16, 71-96.

Cadieux, Louise. (2007). "Succession in Small and Medium-Sized Family Business: Toward a Typology of Predecessor Roles during and After Instatement of the Successor". Family Business Review, 20 (2), 95-109.

Chirico, Francisco y Salvato, Carlos. (2008). "Knowledge Integration and Dynamic Organizational Adaptation in Family Firms". Family Business Review, $21(2), 169-181$.

Christman, James; Chua, Jess; Sharma Pramodita. (1998). "Important Atributtes of Succesors in Family Business: An Exploratory Study". Family Business Review, 11(1), 19-34.

Chua, Jess; Christman, James; Sharma, Pramodita. (1999). "Defining the Family Business by Behavior". Entrepreneurship Theory and Practice, 23(4), 19-39.

Davis, Peter. (1983). "Realizing the Potential of the Family Business." Organizational Dynamics, 12(1), 47-56.

De Massis, Alfredo; Chua, Jess; Chrisman, James. (2008). "Factors Preventing Intra-Family Succession". Family Business Review, 21(2), 183-199.

Diericx, Ingemar. y Cool, Karel. (1989). "Asset Stock Accumulation and the Sustainability Competitive advantage". Management Science, Vol. 35, 15041511.

Dumas, Colette; Dupuis, Joan Pierre; Richer, Francine; St.-Cyr, Louise. (1995). "Factors that influence the next generation's decision to take over the 
family farm". Family Business Review, 8(2), 99-120.

Dunn, Barbara (1999). “The Family Factor: The Impact of Family Relationship Dynamics on Business-Owning Families during Transitions". Family Business Review, 12(1), 41-60.

Drennan, Judy; Kennedy, Jessica; Renfrow, Patty. (2005). "Impact of childhood experiences on the development of entrepreneurial intentions". The International Journal of Entrepreneurship and Innovation, 6(4), 231-238.

Eckrich,Christopher y Loughead, Teri. (1996). Effects of Family Business Membership and Psychological separation on the career Development of Late Adolescents. Family Business Review, 9(4), 369-386.

Eisenhardt, Kathleen M. (1989). Building Theories from Case Study Research. Academy of Management Review, 14(4), 532-550.

García, María Ercilia y López, Jordi. (2001). "ATaxonomy of founders based on Values: The root of family business heterogeneity". Family Business Review, 14(3), 209-227.

García, María Ercilia. (2001). "Los Valores del Fundador y su influencia en la empresa familiar en Galicia". Tesis de Doctorado, 390 p., Universidad Autónoma de Barcelona, España.

García, María Ercilia y López, Jordi. (2003). "Coherence between values and successor socialization: facilitating family business continuity". Working paper, WPS12, Julio, published by the chair of family business, IESE.

Gimeno, Alberto; Álvarez, José Luis; Baulenas, Gemma; Nieto, Eugenia; Servalós, Ricard. (2008). Programa Gestión Avanzada de la Empresa Familiar. Executive Education, ESADE Business School. Barcelona, España. Mayo 26, 27, 28.
Grant, Robert M. (1996a). "Toward a Knowledge-Based Theory of the Firm". Strategic Management Journal, Vol.17, 109-122.

Grant, Robert M. (1996b). Dirección Estratégica. Conceptos, técnicas y aplicaciones. Civitas, Madrid.

Goldberg, Steven D. (1996). "Effective Successors in Family Owned Business: Significant Elements". Family Business Review, 9(2), 185-197.

Handler, Wendy C. y Kram, Kathy E. (1988). "Succession in Family Firms: The Problem of Resistance". Family Business Review, 1(4), 361-381.

Harvey, Michael y Evans, Rodney E. (1994). "The Impact of Timing and Mode of Entry on Successor Development and Successful Succession", Family Business Review, 7(1), 221-236.

Haberman, Heather y Danes, Sharon. (2007). "Father-Daughter and Father-Son Family Business Management Transfer Comparison: Family FIRO Model Application". Family Business Review, 20(2), 163-184.

Habbershon, Timothy G. y Pistrui, Joseph. (2002). "Enterprising Families Domain: Family-Influenced Ownership Groups in Pursuit of Transgenerational Wealth". Family Business Review, 15(3), 223-238.

Howorth, Carole y Assaraf, Zahra. (2001). "Family business succession in Portugal: An examination of case studies in the furniture industry". Family Business Review, 14(3), 231-244.

Lambrech, Joan. (2005). "Multigenerational Transition in Family Business: A New Explanatory Model". Family Business Review, 18(4), 267-282.

Lansberg Iván y Astrachan, Joseph. (1994). "Influence of Family Relationships on Succession Planning and Training: The Importance of Mediating Factors". Family Business Review, 7(1), 39-59. 
Longenecker, Justin y Schoen, John. (1978). "Management succession in the family business". Journal of Small Business Management, 16 (3), 1-6.

Lozano, Melquicedec. (2003). "Las relaciones intrafamiliares en la empresa familiar". Revista Pensamiento y Gestión de la facultad de Administración de Empresas de La Universidad del Norte, No. 15. Barranquilla, Colombia.

Lozano, Melquicedec. (2004). "Cómo perciben los estudiantes la educación empresarial en la secundaria". Memorias III Conferencia Internacional de investigación en Entrepreneurship en América Latina. Pontificia Universidad Católica de Río. Noviembre 11, 12, 13 de 2005. Río de Janeiro, Brasil.

Lozano, Melquicedec. (2006). "La relación intrafamiliar en entornos empresariales. Incursión a un modelo de diagnóstico". Revista Estudios Gerenciales, Facultad de Ciencias Administrativas y Económicas de la Universidad Icesi, 22 (100), 125-150.

Lozano, Melquicedec. (2008). "Elementos del Proceso de Formación de Descendientes antes de su Vinculación a la Empresa familiar. Un Estudio de casos Colombianos". Cuadernos de Administración, Facultad de Ciencias Económicas y Administrativas de la Pontificia Universidad Javeriana, 21 (37), 243-268.

Lozano, Melquicedecy Urbano, David. (2008). "La Vinculación de Descendientes a la Empresa Familiar. Un Estudio de casos colombianos". Revista Estudios Gerenciales, Facultad de Ciencias Administrativas y Económicas de la Universidad Icesi, 24(109), 37-63.

Lumpkin, George T.; Martin, Wendy; Vaughn, Marsha (2008). "Family Orientation: Individual Level Influences on Family Firm Outcomes". Family Business Review, 21(2), 127-138.
Marshall, Catherine y Rossman, Gretchen B. (1995). "Designing Qualitative Research", 2a. ed., Thousand Oaks, CA: Sage Publications..

Meyer, John y Herscovitch, Lynne. (2001). "Commitment in the workplace toward a general mode". Human Resource Management Review, 11, 299-326.

Miles, Matthew y Huberman, Michael. (1994). Qualitative Data analysis: A Sourcebook of New Methods. California: Sage Publications.

Moreno, Ana y Casillas, José. (2008). "Entrepreneurial Orientation and Growth of SMEs: A Causal Model". Entrepreneurship, Theory and Practice, mayo, 507-528.

Morris, Michael; Williams, Roy; Nel, Deon. (1996). "Factors influencing family business succession". International Journal of Entrepreneurial Behaviour \& Research. 2(3), 68-81.

Morris, Michael; Williams, Roy; Allen, Jeffrey; Avila, Ramón. (1997), "Correlates of success in family business transitions", Journal of Business Venturing, 12(5), 385-401.

Navas, José E. y Guerras, Luis Ángel. (1998). "La Dirección Estratégica de la Empresa. Teoría y Aplicaciones". Editorial Civitas, segunda edición. Madrid, España.

Neubauer, Freud. y Lank, Alden. (1999). La empresa Familiar, Cómo dirigirla para que perdure. Deusto. Bilbao, España.

Perry, Chad. (1998). Processes of a case study methodology for postgraduate research in marketing. European Journal of Marketing, 32(9/10), 785-802.

Peteraf, Margaret. (1993). "The Cornerstones of Competitive Advantage: A Resource-Base View". Strategic Management Journal, Vol.14, 179191. 
Sharma, Pramodita y Rao, Srinivas. (2000). Succesor Attributes in Indian and Canadian Family Fims: A Comparable Study". Family Business Review, 13(4), 313-330.

Sharma, Pramodita; Chrisman, James; Pablo, Amy; Chua, Jess. (2001). "Determinants of initial satisfaction with the succession process in family firms: A conceptual model". Entrepreneurship Theory and Practice, 25(3), 17-33.

Sharma, Pramodita; Chrisman, James; Chua, Jess. (2003a). "Predictors of satisfaction with the Succession Process in Family Firms". Journal of business Venturing, 18(5), 667-687.

Sharma, Pramodita; Chrisman James; Chua, Jess. (2003b). "Succession Planning as Planned Behavior: Some Empirical Results", Family Business Review, 16(1), 1- 15.

Sharma, Pramodita e Irving, Gregory. (2005). Four basis of family business successor commitment: antecedents and consequences. Entrepreneurship Theory and Practice, January, 13-21.

Stafford, Kathryn; Duncan, Karen; Dane, Sharon; Winter, Mary (1999). "A Research Model of Sustainable Family Business", Family Business Review, 12(3), 197-208.

Stavrou, Eleni y Swiercz, Paul Michael. (1998). "Securing the Future of the Family Enterprise: A Model of Offspring Intentions to Join the Business". Entrepreneurship Theory and Practice, 23(2), 19-39.

Stavrou, Eleni. (1999). "Succession in Family Businesses: Exploring the Effects of Demographic Factors on Offspring Intentions to Join and Take Over the Business". Journal of Small Business Management, 37(3), 43-61.

Strauss, Anselm y Corbin, Juliet. (1990). Basics of Qualitative Research:
Grounded Theory Procedures and Techniques (p. 270.). Londres: Sage.

Sorenson, Ritch. (2000). "The Contribution of Leadership Style and Practices to Family and Business Success". Family Business Review, 13(3), 183200.

Turner, Alicia. (1995). "Developing Leadership in the Successor Generation". Family business review, 8(3), 201-209.

Upton, Nancy; Teal, Elisabeth; Felan, Joe T. (2001). "Strategic and Business Planning Practices of fast Growth Family Firms". Journal of small business management, 39(1), 60-72.

Ussman, Ana María. (1994). "A transferencia de geracao na direccao das empresas familiares em Portugal". Doctoral Dissertation. Universidade Da Beira Interior, Portugal. Universidad Autónoma de Barcelona, España.

Venter, Elmarie; Boshoff, Christo; Maas, Gideon. (2005). "The influence of Successor-Related Factors on the Succession Process in Small and Medium-Sized Family Businesses". Family Business Review, 18(4), 283-303.

Ward, John (1994). "Cómo desarrollar la empresa familiar". Ediciones el Ateneo, Buenos Aires, Argentina.

Ward, John. (1997). "Growing the Family Business: Special Challenges and Best Practices". Family Business Review, 10(4), 323-337.

Ward, John y Dolan, Christina. (1998). "Defining and Describing Family Business Ownership Configurations". Family Business Review, 11(4), 305-310.

Westhead, Paul. (1997). "Ambitions, external environment and strategic factor differences between family and nonfamily companies". Entrepreneurship and Regional Development, 9(2), 126-157. 
Westhead, Paul y Crowling, Marc. (1998), "Family Firm Research: the Need for a Methodological Rethink". Entrepreneurship Theory and Practice, 23(1), 31-55.

Wernerfelt, Birger. (1984). "A resource-based view of the firm". Strategic Management Journal, Vol. 5, 171-180.

Williams, Roy O. (1992). "Successful Ownership in Business Families". Family Business Review, 5(2), 161-172.

Winter, Mary; Fitzgeral, Margaret; Heck, Ramona; Haynes, George; Danes,
Sharon. (1998). "Revisiting the study of family business: methodological challenges, dilemmas, and alternative approaches". Family business review, 11(3), 239-252.

Wortman Jr., Max. (1994)."Theoretical foundations for family-owned businesses: A conceptual and research based paradigm". Family Business Review, 7(1): 3-27.

Yin, Robert. (1994). Case Study Research. Design and Methods. Sage publications, London. 